ARTIGO ORIGINAL

\title{
Efeitos de diferentes composições de camadas em painéis estruturais de partículas mistas de Eucalyptus badjensis e Pinus spp.
}

\author{
Effects of different layer compositions on structural panels of mixed particles of \\ Eucalyptus badjensis and Pinus spp.
}

\author{
Giuliano Ferreira Pereira' (D), Setsuo Iwakiri ${ }^{1}$ (D), Rosilani Trianoski ${ }^{1}$ (D), Polliana D'angelo Rios ${ }^{2}$ (D), \\ Renan Zunta Raia' ${ }^{1}$ \\ ${ }^{1}$ Universidade Federal do Paraná - UFPR, Curitiba, PR, Brasil \\ Universidade do Estado de Santa Catarina - UDESC, Lages, SC, Brasil
}

\begin{abstract}
Como citar: Pereira, G. F., Iwakiri, S., Trianoski, R., Rios, P. D., \& Raia, R. Z. (2021). Efeitos de diferentes composições de camadas em painéis estruturais de partículas mistas de Eucalyptus badjensis e Pinus spp. Scientia Forestalis, 49(129), e3332. https://doi.org/10.18671/scifor.v49n129.11
\end{abstract}

\begin{abstract}
Resumo
Esta pesquisa teve como objetivo avaliar o potencial de utilização de painéis de partículas mistas para fins estruturais, estudar os efeitos de diferentes composições de camadas e tipos de partículas sobre os painéis, bem como, analisar o potencial da madeira de Eucalyptus badjensis e sua aplicação em conjunto com a madeira de Pinus spp. para produção deste tipo de painel. Foram produzidos painéis de partículas mistas de Eucalyptus badjensis e Pinus spp. com proporções sliver/strand/sliver de 10/80/10, 15/70/15 e 20/60/20, além de painéis testemunha de MDP e OSB. As propriedades físicas e mecânicas dos painéis foram avaliadas conforme os procedimentos recomendados pelas normas EN 323, EN 317, EN 319 e EN 310. Os resultados permitiram concluir que os painéis de partículas mistas possuem potencial de uso para fins estruturais, que a composição 10/80/10 apresenta os melhores resultados para as propriedades físicas e mecânicas estudadas e que a madeira de Eucalyptus badjensis, bem como sua aplicação em conjunto com a madeira de Pinus spp. apresentam potencial para produção de painéis de partículas mistas.
\end{abstract}

Palavras-chave: Strand; Sliver; Resina fenólica.

\begin{abstract}
The objective of this research was to evaluate the potential of using mixed particle boards for structural purposes, to study the effects of different layer compositions and particle types on the boards, as well as to analyze the potential of Eucalyptus badjensis wood and its use with the wood of Pinus spp. for production of this type of panel. Were produced mixed particle panels of Eucalyptus badjensis and Pinus spp. with sliver/strand/sliver ratios of 10/80/10, 15/70/15 and 20/60/20, as well as MDP and OSB control panels. The physical and mechanical properties of the panels were evaluated according to the procedures recommended by EN 323, EN 317, EN 319 and EN 310. The results allowed to conclude that the mixed particle board has potential use for structural purposes, that the composition 10/80/10 presents the best results for the physical and mechanical properties and that Eucalyptus badjensis wood, as well as its use in conjunction with Pinus spp. have potential for mixed particle panels production.
\end{abstract}

Keywords: Strand; Sliver; Phenolic resin.

Fonte de financiamento: Capes/DS.

Conflito de interesse: Nada a declarar.

Autor correspondente: giuliano.giiu@gmail.com

Recebido: 9 abril 2019.

Aceito: 5 dezembro 2019

Editor: Paulo Henrique Müller Silva.

(c) (i) Este é um artigo publicado em acesso aberto (Open Access) sob a licença Creative Commons Attribution, que permite uso, distribuição e reprodução c) em qualquer meio, sem restrições desde que o trabalho original seja corretamente citado. 


\section{INTRODUÇÃO}

O Eucalyptus badjensis Beuzev. \& Welch é uma espécie nativa das regiões montanhosas da Austrália. Por apresentar elevadas taxas de crescimento e resistência à geada, a espécie apresenta potencial para plantios em regiões frias do Brasil (Higa et al., 2002).

A utilização de Eucalyptus badjensis vem sendo estudada para os mais variados produtos de origem florestal, como óleos essenciais, celulose, papel e alguns painéis de madeira (Pereira et al., 2017). Contudo, estudos relacionados à sua utilização para produção de painéis de partículas de madeira ainda são incipientes.

Os painéis de partículas de madeira são materiais compósitos que apresentam uma vasta quantidade de aplicações finais, principalmente relacionadas à indústria moveleira e construção civil, como pisos, portas, forros, entre outros (Marzbani et al., 2015).

Os processos produtivos de painéis particulados de madeira são altamente tecnológicos, com um elevado número de variáveis que interagem entre si (Thoemen et al., 2010). Entre essas variáveis é possível destacar a geometria das partículas, uma vez que sua correta definição é determinante nas propriedades finais e aplicação do produto (Iwakiri, 2005).

$\mathrm{O}$ uso de partículas finas e alongadas geralmente fornece aos painéis melhores resultados de resistência a flexão estática (Chiromito et al., 2016). Por outro lado, partículas menores conferem ao painel melhores propriedades de acabamento superficial (Thoemen et al., 2010).

Na produção de painéis de partículas orientadas (OSB) são utilizadas partículas do tipo strand, enquanto que para produção de painéis de partículas de média densidade (MDP) são empregadas partículas menores do tipo sliver (Thoemen et al., 2010).

Por sua vez, as partículas podem ser dispostas em diferentes quantidades nas camadas. Nesse contexto, diversos estudos vêm sendo realizados, como o de Trianoski et al. (2016), que estudaram painéis OSB de Cryptomeria japonica nas proporções face/miolo/face de 20/60/20 e 30/40/30 e concluíram que a composição de camadas 20/60/20 gerou maior balanceamento das propriedades de flexão estática.

Perante o exposto, o estudo teve como objetivo, avaliar o potencial de utilização de painéis de partículas mistas para fins estruturais, estudar o efeito de diferentes composições de camadas e tipos de partículas sobre as propriedades dos painéis, bem como, analisar o potencial da madeira de Eucalyptus badjensis e sua aplicação em conjunto com a madeira de Pinus spp. para a produção de painéis de partículas mistas.

\section{MATERIAL E MÉTODOS}

\subsection{Coleta e preparação do material}

Para a produção dos painéis foi utilizada madeira de Eucalyptus badjensis, proveniente de povoamento florestal localizado no município de Lages - SC. As árvores foram derrubadas e seccionadas em toras de $2 \mathrm{~m}$. Para determinação da massa específica básica da madeira, foram retirados a cada $2 \mathrm{~m}$, discos de $5 \mathrm{~cm}$ e, de cada disco, foram obtidas quatro cunhas para a realização dos ensaios.

Posteriormente, as toras foram transportadas até uma serraria localizada no município Otacílio Costa - SC, onde foram desdobradas em tábuas com a espessura de uma polegada, para então serem encaminhadas ao Laboratório de painéis de madeira da UFPR em Curitiba - PR.

Por sua vez, a madeira de Pinus spp. foi adquirida de um estabelecimento comercial de Curitiba - PR na forma de tábuas de uma polegada. Dessas tábuas foram retirados, de forma aleatória, corpos-de-prova para a determinação da massa específica básica.

Para a geração de partículas, as tábuas de Eucalyptus badjensis e de Pinus spp. foram seccionadas em peças menores e em seguida encaminhadas a um picador, onde foram transformadas em partículas do tipo strand, as quais foram classificadas em peneira com abertura de 9,52 mm para retirada de partículas menores a esta dimensão. Os Strands produzidos apresentavam dimensões médias de $74,5 \mathrm{~mm} \times 17,0 \mathrm{~mm} \times 0,8 \mathrm{~mm}$. 
Em um moinho de martelo parte das partículas strand foram transformadas em partículas do tipo sliver, sendo posteriormente conduzidas a um classificador automático para a remoção dos finos, utilizando peneiras de 8 e 30 mesh, com aberturas de 2,36 mm e 0,6 $\mathrm{mm}$, respectivamente. As partículas sliver produzidas apresentavam dimensões médias de $11,0 \mathrm{~mm} \times 1,5 \mathrm{~mm} \times 0,5 \mathrm{~mm}$.

\subsection{Avaliações}

A massa específica básica das madeiras de Eucalyptus badjensis e Pinus spp. foi calculada de acordo com os procedimentos recomendados pela norma NBR 7190 (Associação Brasileira de Normas Técnicas, 1997).

O experimento foi conduzido em delineamento inteiramente casualizado (DIC). Os painéis foram produzidos conforme o plano experimental apresentando na Tabela 1 e, para cada tratamento, foram produzidos 3 painéis.

Para a produção dos painéis, todas as partículas foram secas em estufa ao teor de umidade médio de $4 \%$ e conduzidas a uma encoladeira para aplicação de $1 \%$ de parafina e $6 \%$ de resina fenol-formaldeído, com teor de sólidos de 53\% e viscosidade de 492 cP.

Tabela 1. Delineamento experimental utilizado para a produção dos painéis testemunha e painéis estruturais de partículas mistas produzidos com Pinus spp. e Eucalyptus badjensis

\begin{tabular}{cccc}
\hline Tratamento & Camadas externas & Camada interna & DC \\
\hline 1 & Slivers Pinus spp. & - & Camada única \\
2 & Slivers E. badjensis & - & Camada única \\
3 & * Strands Pinus spp. & Strands Pinus spp. & $20 / 60 / 20$ \\
4 & *Strands E. badjensis & Strands E. badjensis & $20 / 60 / 20$ \\
5 & Slivers Pinus spp. & Strands E. badjensis & $20 / 60 / 20$ \\
6 & Slivers Pinus spp. & Strands E. badjensis & $15 / 70 / 15$ \\
7 & Slivers Pinus spp. & Strands E. badjensis & $10 / 80 / 10$ \\
8 & Slivers E. badjensis & Strands E. badjensis & $20 / 60 / 20$ \\
9 & Slivers E. badjensis & Strands E. badjensis & $15 / 70 / 15$ \\
10 & Slivers E. badjensis & Strands E. badjensis & $10 / 80 / 10$
\end{tabular}

DC: Disposição de camadas, *Painéis do tipo OSB, Painéis testemunha (tratamentos 1 a 4) produzidos com massa específica nominal de $0,700 \mathrm{~g} / \mathrm{cm}^{3}$, Painéis estruturais de partículas mistas (tratamentos 5 a 10) produzidos com massa específica $0,900 \mathrm{~g} / \mathrm{cm}^{3}$ nas camadas externas e $0,700 \mathrm{~g} / \mathrm{cm}^{3}$ na camada interna com partículas dispostas de forma aleatória em todas as camadas.

As partículas foram encaminhadas para a formação do colchão e pré-prensagem em uma caixa formadora com dimensões de $48 \mathrm{~cm} \times 48 \mathrm{~cm}$. A espessura nominal dos painéis foi estabelecida com espaçador de $1,3 \mathrm{~cm}$.

Posteriormente, o colchão de partículas foi prensando durante 8 minutos, com temperatura de $180^{\circ} \mathrm{C}$ e pressão específica de $4,0 \mathrm{MPa}$. Para os painéis testemunha foi utilizada a massa específica nominal de $0,700 \mathrm{~g} / \mathrm{cm}^{3}$ para todas as camadas. Já para os painéis estruturais de partículas mistas, a massa específica nominal foi de $0,700 \mathrm{~g} / \mathrm{cm}^{3}$ para camada interna e $0,900 \mathrm{~g} / \mathrm{cm}^{3}$ para as camadas externas. As massas específicas nominais das diferentes camadas foram calculadas com base no peso seco e dimensões do painel.

Após a prensagem, os painéis foram esquadrejados e acondicionados à temperatura de $20 \pm 2^{\circ} \mathrm{C}$ e umidade relativa de $65 \pm 5 \%$ até a sua estabilização ao teor de umidade médio de $12 \%$.

Para avaliação das propriedades físicas e mecânicas dos painéis, foram retirados de cada painel, cinco corpos-de-prova para ensaios de massa específica aparente, cinco para 
absorção de água e inchamento em espessura após 2 e 24 horas de imersão em água, quatro para flexão estática e cinco para tração perpendicular à superfície. Os ensaios foram realizados conforme os procedimentos descritos nas normas europeias, EN 323, EN 317, EN 319, EN 310, respectivamente (European Committee for Standardization, 1993b, 2002a, 2002b, 2002c).

Os resultados foram submetidos aos testes de Kolmorogorov-Smirnov e Bartlett para a verificação da normalidade e homogeneidade dos dados, respectivamente. $O$ efeito das camadas e espécies foi avaliado por meio de análise fatorial (3×2), além da comparação com os painéis testemunha correspondentes a cada espécie utilizada na camada externa. Em função da análise dos dados não demonstrar interação entre as variáveis espécie e percentual de camadas em nenhuma das propriedades, os dados foram analisados separadamente.

Pelo fato de os tratamentos apresentarem massas específicas nominais distintas, foram realizadas análises de correlação entre a massa específica dos painéis e cada uma das propriedades.

Para os tratamentos em que foi constatada correlação positiva foi realizada a análise de covariância com as médias ajustadas à massa específica dos painéis. Todos os tratamentos foram submetidos ao teste de Tukey ao nível de confiança de $95 \%$.

Em função dos painéis de partículas mistas apresentarem finalidade estrutural e serem produzidos com partículas strand e sliver dispostas de forma aleatória, para a análise dos dados, os valores médios foram comparados com os requisitos mínimos estipulados pelas normas EN 310 (European Committee for Standardization, 2002a) de MDP, EN 312 (European Committee for Standardization, 2003) de OSB, na categoria OSB 3, para painéis estruturais para uso em condições úmidas e CSA 0417 (Canadian Standards Association, 1993) de waferboard e OSB, na categoria R1 para painéis com partículas dispostas de forma aleatória.

\section{RESULTADOS E DISCUSSÃO}

\subsection{Massa específica básica da madeira}

Os valores médios de massa específica básica foram de 0,505 g/ $\mathrm{cm}^{3}$ para Eucalyptus badjensis e de $0,435 \mathrm{~g} / \mathrm{cm}^{3}$ para Pinus spp., sendo os valores considerados adequados para a produção de painéis particulados. De acordo com Maloney (1993), madeiras com massa específica básica de até $0,550 \mathrm{~g} / \mathrm{cm}^{3}$ são as mais recomendadas para produção de painéis de partículas, pois atingem a razão de compactação mínima de 1.3, necessária para que ocorra a compactação das partículas e densificação do painel até a espessura final.

\subsection{Propriedades físicas dos painéis}

Para massa específica aparente, os valores médios obtidos foram de $0,689 \mathrm{~g} / \mathrm{cm}^{3}$ até $0,759 \mathrm{~g} / \mathrm{cm}^{3}$ e apresentaram uma pequena variação em relação a massa específica nominal (Tabela 2).

De acordo com Iwakiri et al. (2012), as diferenças entre massa específica nominal e massa específica aparente podem ser atribuídas às perdas de materiais durante a formação dos painéis e ao retorno em espessura após a prensagem à quente.

De acordo com a classificação estabelecida pela NBR 14810 (Associação Brasileira de Normas Técnicas, 2006), com exceção dos painéis de partículas mistas de Pinus spp. na composição 20/60/20, os demais tratamentos podem ser classificados como painéis de média massa específica $\left(0,550 \mathrm{~g} / \mathrm{cm}^{3}\right.$ até $\left.0,750 \mathrm{~g} / \mathrm{cm}^{3}\right)$.

Já para razão de compactação, os valores médios obtidos variaram de 1,375 até 1,648, desse modo, todos os tratamentos atingiram o valor mínimo de 1,3 recomendado por Maloney (1993), indicando bom contato entre as partículas para os diferentes tratamentos. 
Tabela 2. Valores médios de massa específica aparente, massa específica nominal e razão de compactação dos painéis testemunha e painéis estruturais de partículas mistas produzidos com Eucalyptus badjensis e Pinus spp.

\begin{tabular}{|c|c|c|c|}
\hline Tratamento & $\begin{array}{c}\text { MEA } \\
\left(\mathrm{g} / \mathrm{cm}^{3}\right)\end{array}$ & $\begin{array}{l}\text { ME nominal } \\
\qquad\left(\mathrm{g} / \mathrm{cm}^{3}\right)\end{array}$ & $\begin{array}{c}\text { Razão de } \\
\text { compactação }\end{array}$ \\
\hline MDP Pinus spp. & $\begin{array}{l}0,689 a \\
(5,21)\end{array}$ & 0,700 & $\begin{array}{c}1,604 \mathrm{~cd} \\
(5,89)\end{array}$ \\
\hline MDP E. bad & $\begin{array}{c}0,692 a b \\
(6,59)\end{array}$ & 0,700 & $\begin{array}{c}1,375 a \\
(7,97)\end{array}$ \\
\hline OSB Pinus spp. & $\begin{array}{c}0,717 a b c \\
(8,80)\end{array}$ & 0,700 & $\begin{array}{l}1,648 d \\
(7,72)\end{array}$ \\
\hline & $0,709 a b c$ & & 1,439ab \\
\hline OSB E. bad & $(5,09)$ & 0,700 & $(5,93)$ \\
\hline $\begin{array}{c}\text { PEPM } \\
\text { Pinus spp. } 20 / 60 / 20\end{array}$ & $\begin{array}{l}0,759 c \\
(6,70)\end{array}$ & 0,760 & $\begin{array}{c}1,593 \mathrm{~cd} \\
(5,77)\end{array}$ \\
\hline $\begin{array}{c}\text { PEPM } \\
\text { Pinus spp. 15/70/30 }\end{array}$ & $\begin{array}{c}0,737 a b c \\
(5,69)\end{array}$ & 0,740 & $\begin{array}{l}1,561 \mathrm{c} \\
(5,13)\end{array}$ \\
\hline $\begin{array}{c}\text { PEPM } \\
\text { Pinus spp. } 10 / 80 / 10\end{array}$ & $\begin{array}{c}0,713 a b c \\
(5,34)\end{array}$ & 0,720 & $\begin{array}{c}1,506 \mathrm{bc} \\
(5,03)\end{array}$ \\
\hline $\begin{array}{c}\text { PEPM } \\
\text { E. bad } 20 / 60 / 20\end{array}$ & $\begin{array}{c}0,749 b c \\
(6,00)\end{array}$ & 0,760 & $\begin{array}{c}1,482 \mathrm{abc} \\
(6,76)\end{array}$ \\
\hline $\begin{array}{c}\text { PEPM } \\
\text { E. bad } 15 / 70 / 15\end{array}$ & $\begin{array}{c}0,734 a b c \\
(6,51)\end{array}$ & 0,740 & $\begin{array}{c}1,416 a b \\
(6,20)\end{array}$ \\
\hline $\begin{array}{c}\text { PEPM } \\
\text { E. bad } 10 / 80 / 10\end{array}$ & $\begin{array}{c}0,720 \mathrm{abc} \\
(8,80)\end{array}$ & 0,720 & $\begin{array}{l}1,401 a \\
(9,84)\end{array}$ \\
\hline
\end{tabular}

Médias seguidas de letras iguais na mesma coluna não diferem entre si pelo teste de Tukey ao nível de $95 \%$ de probabilidade. Coeficiente de variação, em porcentagem, entre parênteses; MEA: Massa específica aparente; ME nominal: Massa específica nominal; E bad: Eucalyptus badjensis. PEPM: Painel estrutural de partículas mistas.

Para absorção de água após 2 e 24 horas, dentre os painéis com Pinus spp. na camada externa, os painéis estruturais de partículas mistas apresentaram os menores valores médios, sendo estatisticamente diferentes dos painéis OSB e MDP para absorção de água após 2 e 24 horas de imersão (Tabela 3).

Este fato pode ser explicado pela utilização de strands de Eucalyptus badjensis na camada interna dos painéis mistos, resultando em painéis com menor razão de compactação, compostos de menor quantidade de partículas e área superficial das mesmas, proporcionando maior estabilidade dimensional dos painéis.

Para os painéis produzidos com Eucalyptus badjensis nas camadas externas, nos ensaios de absorção de água após 2 e 24 horas de imersão, os painéis de partículas mistas apresentaram melhores resultados que os painéis MDP. Já, em comparação com os painéis 
OSB, os painéis estruturais de partículas mistas apresentaram valores médios semelhantes para o ensaio de absorção de água após 2 horas de imersão e inferiores ou semelhantes para o ensaio de absorção de água após 24 horas de imersão.

Tabela 3. Valores médios de absorção de água após 2 e 24 horas de imersão para painéis de mesma espécie na camada externa.

\begin{tabular}{ccccccc}
\hline \multirow{2}{*}{ Tratamento } & \multicolumn{2}{c}{ AA2h (\%) } & & \multicolumn{2}{c}{ AA24h (\%) } \\
\cline { 2 - 3 } \cline { 5 - 6 } & Pinus & E bad & & Pinus & E bad \\
\hline \multirow{2}{*}{ MDP } & $29,49 \mathrm{~b}$ & $17,04 \mathrm{~b}$ & & $63,41 \mathrm{c}$ & $56,67 \mathrm{c}$ \\
& $(15,09)$ & $(16,78)$ & & $(17,56)$ & $(18,74)$ \\
OSB & $28,77 \mathrm{~b}$ & $12,52 \mathrm{a}$ & & $63,37 \mathrm{c}$ & $40,45 \mathrm{a}$ \\
& $(16,43)$ & $(18,16)$ & & $(18,74)$ & $(13,21)$ \\
PEPM & $24,43 \mathrm{a}$ & $16,10 \mathrm{ab}$ & & $58,80 \mathrm{~b}$ & $47,11 \mathrm{~b}$ \\
$\mathbf{2 0 / 6 0 / 2 0}$ & $(26,01)$ & $(20,83)$ & & $(12,82)$ & $(19,39)$ \\
PEPM & $24,47 \mathrm{a}$ & $13,14 \mathrm{a}$ & & $42,00 \mathrm{a}$ & $46,21 \mathrm{~b}$ \\
$\mathbf{1 5 / 7 0 / 1 5}$ & $(17,18)$ & $(20,76)$ & & $(12,10)$ & $(21,70)$ \\
PEPM & $21,62 \mathrm{a}$ & $12,81 \mathrm{a}$ & & $44,99 \mathrm{~b}$ & $44,03 \mathrm{ab}$ \\
$\mathbf{1 0 / 8 0 / 1 0}$ & $(18,09)$ & $(25,04)$ & & $(11,82)$ & $(19,46)$ \\
\hline
\end{tabular}

Médias seguidas de letras iguais na mesma coluna não diferem entre si e médias pelo teste de Tukey ao nível de 95\% de probabilidade. Coeficiente de variação, em porcentagem, entre parênteses. AA2h: Absorção de água após 2 horas; AA24h: Absorção de água após 24 horas; Pinus: Pinus spp.; E bad: Eucalyptus badjensis; PEPM: Painel estrutural de partículas mistas.

Estes resultados podem ser explicados pelos tipos das partículas utilizadas no estudo, uma vez que os painéis produzidos com maior quantidade de partículas do tipo strand apresentaram os melhores valores médios para absorção de água após 2 e 24 horas de imersão. Conforme Moslemi (1974), o uso de partículas maiores ocasiona uma encolagem mais eficiente, em função de apresentarem menor área superficial específica, por conseguinte, maior disponibilidade de adesivo por área.

Para o inchamento em espessura, dentre os painéis com Pinus spp. nas camadas externa, os painéis estruturais de partículas mistas apresentaram os melhores valores médios, tendo como exceção a composição 20/60/20, em que apresentou valor médio estatisticamente semelhante ao obtido para o tratamento de MDP no ensaio de inchamento em espessura após 24 de imersão (Tabela 4).

Para os painéis com Eucalyptus badjensis na camada externa, nos ensaios de inchamento em espessura após 2 e 24 horas de imersão, os painéis estruturais de partículas mistas apresentaram valores médios semelhantes aos painéis OSB e menores que os obtidos para os painéis MDP.

De forma geral, os painéis estruturais de partículas mistas apresentaram os melhores valores médios para estabilidade dimensional entre os painéis produzidos com Pinus spp. na camada externa. Este fato pode ser explicado pela utilização de strands de Eucalyptus badjensis na camada interna nesses painéis, tendo em vista que esta espécie apresenta massa específica maior que a da madeira Pinus spp.

Segundo Iwakiri (2005), espécies com maior massa específica básica, em decorrência da menor liberação das tensões de compressão ocorridas na prensagem, geralmente, apresentam menores valores de inchamento em espessura quando comparados com espécies de menor massa específica.

Em comparação com os requisitos normativos, para inchamento em espessura após 2 horas, apenas os painéis MDP e OSB de Pinus spp. não obtiveram valores médios correspondentes aos recomendados pela norma CSA 0437. 
Tabela 4. Valores médios de inchamento em espessura após 2 e 24 horas de imersão para painéis de mesma espécie na camada externa.

\begin{tabular}{cccccc}
\hline & \multicolumn{2}{c}{ IE2h (\%) } & & \multicolumn{2}{c}{ IE24h (\%) } \\
\cline { 2 - 3 } \cline { 5 - 6 } Tratamento & Pinus & E bad & & Pinus & E bad \\
\hline MDP & $10,57 \mathrm{~b}$ & $07,12 \mathrm{~b}$ & & $24,85 \mathrm{~b}$ & $25,12 \mathrm{~b}$ \\
& $(21,84)$ & $(23,37)$ & & $(18,90)$ & $(17,44)$ \\
OSB & $13,34 \mathrm{~b}$ & $04,85 \mathrm{a}$ & & $33,07 \mathrm{c}$ & $09,15 \mathrm{a}$ \\
PEPM & $(20,36)$ & $(20,72)$ & & $(18,91)$ & $(17,55)$ \\
20/60/20 & $09,99 \mathrm{a}$ & $04,51 \mathrm{a}$ & & $23,04 \mathrm{~b}$ & $15,24 \mathrm{a}$ \\
PEPM & $(23,13)$ & $(21,59)$ & & $(26,55)$ & $(27,12)$ \\
15/70/15 & $07,36 \mathrm{a}$ & $04,09 \mathrm{a}$ & & $11,60 \mathrm{a}$ & $13,82 \mathrm{a}$ \\
PEPM & $(27,29)$ & $(25,61)$ & & $(24,28)$ & $(25,49)$ \\
10/80/10 & $06,59 \mathrm{a}$ & $03,85 \mathrm{a}$ & & $10,53 \mathrm{a}$ & $13,39 \mathrm{a}$ \\
CSA0437* & $(22,82)$ & $(16,05)$ & $(13,16)$ & $(13,16)$ \\
EN300** & & 10,00 & & & 15,00
\end{tabular}

Médias seguidas de letras iguais na mesma coluna não diferem entre si e médias pelo teste de Tukey ao nível de 95\% de probabilidade. Coeficiente de variação, em porcentagem, entre parênteses. IE2h: Inchamento em espessura após 2 horas; IE24h: Inchamento em espessura após 24 horas; Pinus: Pinus spp. E bad: Eucalyptus badjensis; PEPM: Painel estrutural de partículas mistas. *Categoria R1: Painéis com partículas dispostas de forma aleatórias, **OSB 3: Painéis estruturais para uso em condições úmidas.

Por outro lado, para inchamento em espessura em 24 horas os tratamentos que atenderam aos requisitos estabelecidos pela EN 300 foram os painéis OSB de Eucalyptus badjensis e os painéis estruturais de partículas mistas nas composições 10/80/10 e 15/70/15, tanto para os painéis de Eucalyptus badjensis, quanto para os de Pinus spp na camada externa.

Comparando os painéis produzidos com Eucalyptus badjensis e Pinus spp. na camada externa. Os painéis produzidos com Eucalyptus badjensis apresentaram menores valores médios que os produzidos com partículas de Pinus spp. nos ensaios de absorção de água e inchamento em espessura após 2 horas de imersão (Tabela 5).

Tabela 5. Valores médios de absorção de água e inchamento em espessura para painéis estruturais de partículas mistas produzidos com diferentes espécies na camada externa.

\begin{tabular}{ccccc}
\hline Espécie & AA2h (\%) & AA24h (\%) & IE2h (\%) & IE24h (\%) \\
\hline Pinus spp. & $24,04 \mathrm{~b}$ & $47,53 \mathrm{a}$ & $7,85 \mathrm{~b}$ & $14,46 \mathrm{a}$ \\
& $(18,92)$ & $(15,78)$ & $(23,83)$ & $(26,86)$ \\
E. bad & $14,68 \mathrm{a}$ & $46,30 \mathrm{a}$ & $4,18 \mathrm{a}$ & $14,38 \mathrm{a}$ \\
& $(18,58)$ & $(16,13)$ & $(21,85)$ & $(21,36)$ \\
CSA0437* & & & 10,00 & \\
EN 300** & & & & 15,00
\end{tabular}

Médias seguidas de letras iguais na mesma coluna não diferem entre tratamentos da mesma espécie pelo teste de Tukey ao nível de 95\% de probabilidade. Coeficiente de variação, em porcentagem, entre parênteses. AA2h: Absorção de água após 2 horas; AA24h: Absorção de água após 24 horas; IE2h: Inchamento em espessura após 2 horas; IE24h: Inchamento em espessura após 24 horas. E bad: Eucalyptus badjensis. *Categoria R1: Painéis com partículas dispostas de forma aleatórias, **OSB 3: Painéis estruturais para uso em condições úmidas. 
Este resultado pode ser atribuído à maior massa específica da madeira de Eucalyptus badjensis, que resulta em painéis com menor razão de compactação e, desse modo, menor quantidade de partículas e área superficial das mesmas, proporcionando maior estabilidade dimensional aos painéis.

Outrossim, não foi constatada diferença estatistica significativa entre os panéis produzidos com Eucalyptus badjensis e Pinus spp. para os ensaios de absorção de água e inchamento em espessura após 24 horas. Indicando que não houve influência significativa entre as espécies estudadas sobre estas propriedades.

Em relação aos requisitos normativos, tanto os painéis de Eucalyptus badjensis, quanto os de Pinus spp. na camada externa, atenderam aos valores máximos para inchamento em espessura após 2 e 24 horas de imersão em água estabelecidos pela CSA 0437 e EN 300, respectivamente (Canadian Standards Association, 1993; European Committee for Standardization, 2006).

Os valores médios obtidos para os painéis com diferentes percentuais de camadas foram estatísticamente semelhantes para absorção de água e inchamento em espessura após 2 horas de imersão em água (Tabela 6).

Tabela 6. Valores médios de absorção de água e inchamento em espessura para painéis produzidos com diferentes composições de camadas

\begin{tabular}{ccccc}
\hline Composição & AA2h (\%) & AA24h (\%) & IE2h (\%) & IE24h (\%) \\
\hline PEPM & $20,64 a$ & $52,31 b$ & $6,53 a$ & $19,20 b$ \\
20/60/20 & $(20,74)$ & $(12,86)$ & $(27,92)$ & $(21,72)$ \\
PEPM & $18,50 a$ & $46,02 a$ & $5,69 a$ & $13,20 a$ \\
15/70/10 & $(24,94)$ & $(16,14)$ & $(26,22)$ & $(29,55)$ \\
PEPM & $17,81 a$ & $43,27 a$ & $5,36 a$ & $11,90 a$ \\
10/80/10 & $(16,02)$ & $(18,02)$ & $(19,75)$ & $(19,84)$ \\
CSA0437* & & & 10,00 & \\
EN 300** & & & & 15,00
\end{tabular}

Médias seguidas de letras iguais na mesma coluna não diferem estatisticamente entre si pelo teste de Tukey ao nível de 95\% de probabilidade. Coeficiente de variação, em porcentagem, entre parênteses. AA2h: Absorção de água após 2 horas; AA24h: Absorção de água após 24 horas; IE2h: Inchamento em espessura em 2 horas; IE24h: Inchamento em espessura em 24 horas, PEPM: Painel estrutural de partículas mistas. *Categoria R1: Painéis com partículas dispostas de forma aleatórias, **OSB 3: Painéis estruturais para uso em condições úmidas.

Nos ensaios de absorção de água e inchamento em espessura após 24 horas de imersão em água, as composições de camadas com 10/80/10 e 15/70/15 (sliver/strand/sliver) apresentaram médias estatisticamente inferiores em compração aos painéis produzidos com composição de 20/60/20.

A redução da absorção de água e inchamento em espessura dos painéis com maior percentual de partículas do tipo strand na camada interna, pode ser atribuída à sua menor área superficial e consequente aumento na disponibilidade de resina entre as partículas.

Em relação aos requisitos normativos, todas as três composições atenderam aos valores estabelecidos pela CSA 0437 para inchamento em espessura após 2 horas de imersão. Para o inchamento em espessura após 24 horas, os painés compostos com relação 10/80/10 e 15/70/15 atenderam aos requistos estabelecidos pela EN 300.

\subsection{Propriedades mecânicas dos painéis}

Para os módulos de elasticidade (MOE) e de ruptura (MOR), observa-se que os painéis estruturais de partículas mistas apresentaram valores médios semelhantes ou maiores que os obtidos para os painéis MDP, tanto para os painéis de Eucalyptus badjensis, quanto para os de Pinus spp. na camada externa. 
Por outro lado, em comparação aos painéis OSB, tanto para o módulo de elasticidade, quanto para o módulo de ruptura, os valores médios obtidos para os painéis estruturais de partículas mistas foram inferiores ou semelhantes (Tabela 7).

Tabela 7. Valores médios de módulo de elasticidade, módulo de ruptura e tração perpendicular à superfície para painéis produzidos com a mesma espécie na camada externa

\begin{tabular}{|c|c|c|c|c|c|c|}
\hline \multirow{2}{*}{ Tratamento } & \multicolumn{2}{|c|}{ MOE (MPa) } & \multicolumn{2}{|c|}{ MOR (MPa) } & \multicolumn{2}{|c|}{ TP (MPa) } \\
\hline & Pinus & E bad & Pinus & E. bad & Pinus & E bad \\
\hline MDP & $\begin{array}{c}2004,68 d \\
(16,01)\end{array}$ & $\begin{array}{c}1948,63 c \\
(12,54)\end{array}$ & $\begin{array}{r}17,87 c \\
(15,33)\end{array}$ & $\begin{array}{l}14,85 c \\
(15,04)\end{array}$ & $\begin{array}{c}0,62 a \\
(10,79)\end{array}$ & $\begin{array}{c}0,41 c \\
(13,07)\end{array}$ \\
\hline OSB/ para & $\begin{array}{c}5988,22 a \\
(25,30)\end{array}$ & $\begin{array}{c}4706,90 a \\
(25,17)\end{array}$ & $\begin{array}{l}38,36^{a} \\
(13,94)\end{array}$ & $\begin{array}{l}25,71 a \\
(19,26)\end{array}$ & $0,61 a$ & $0,55 a$ \\
\hline OSB/ perp & $\begin{array}{c}3169,22 b \\
(24,94)\end{array}$ & $\begin{array}{c}2280,95 b \\
(23,31)\end{array}$ & $\begin{array}{c}27,86 b \\
(14,63)\end{array}$ & $\begin{array}{c}24,12 a b \\
(15,53)\end{array}$ & $(12,69)$ & $(19,71)$ \\
\hline PEPM & $2205,74 c d$ & $2295,25 b c$ & $19,59 b c$ & $17,89 \mathrm{bc}$ & 0,53ab & $0,46 b c$ \\
\hline $20 / 60 / 20$ & $(14,41)$ & $(17,90)$ & $(16,55)$ & $(16,53)$ & $(14,96)$ & $(14,66)$ \\
\hline PEPM & $2437,40 \mathrm{~cd}$ & $2418,84 b$ & $21,37 b c$ & $19,80 \mathrm{bc}$ & $0,55 a b$ & $0,51 a b$ \\
\hline $15 / 70 / 15$ & $(19,33)$ & $(20,66)$ & $(19,01)$ & $(19,79)$ & $(9,29)$ & $(20,33)$ \\
\hline PEPM & $2850,15 b c$ & $2800,11 b$ & $24,74 b$ & $22,87 b$ & $0,50 b$ & $0,51 a b$ \\
\hline $10 / 80 / 10$ & $(18,20)$ & $(25,30)$ & $(19,07)$ & $(22,48)$ & $(15,49)$ & $(23,66)$ \\
\hline CSA0437* & \multicolumn{2}{|c|}{3040} & \multicolumn{2}{|c|}{16,87} & \multicolumn{2}{|c|}{0,34} \\
\hline EN312 & \multicolumn{2}{|c|}{1800} & \multicolumn{2}{|c|}{13,00} & \multicolumn{2}{|c|}{0,40} \\
\hline EN300para** & \multicolumn{2}{|c|}{3500} & \multicolumn{2}{|c|}{20,00} & \multirow{2}{*}{\multicolumn{2}{|c|}{0,32}} \\
\hline EN300perp** & \multicolumn{2}{|c|}{1500} & \multicolumn{2}{|c|}{10,00} & & \\
\hline
\end{tabular}

Médias seguidas de letras iguais na mesma coluna não diferem estatisticamente entre si pelo teste de Tukey ao nível de 95\% de probabilidade. Coeficiente de variação, em porcentagem, entre parênteses. E. bad: Eucalyptus badjensis, MOE: Módulo de elasticidade; MOR: Módulo de ruptura; TP: Tração perpendicular à superfície. PEPM: Painel estrutural de partículas mistas. *Categoria R1: Painéis com partículas dispostas de forma aleatórias, **OSB 3: Painéis estruturais para uso em condições úmidas. para: sentido paralelo, perp: sentido perpendicular.

Estes resultados demonstram a influência positiva da utilização de partículas do tipo strand nas camadas externas do painel. De acordo com Geimer \& Price (1978) as propriedades de flexão estática de painéis de partículas de madeira aumentam diretamente com o comprimento das partículas, em função da maior razão de esbeltez.

Em relação ao ensaio de tração perpendicular à superfície, os painéis estruturais de partículas mistas foram estatisticamente semelhantes entre si. Nos painéis com Pinus spp. na camada externa, a composição 10/80/10 apresentou valores médios inferiores aos obtidos para os painéis MDP e OSB. Já, as composições 20/60/20 e 15/70/15 apresentaram valores médios semelhantes aos obtidos para os painéis testemunha.

Por sua vez, para os painéis de Eucalyptus badjensis na camada externa, os painéis estruturais de partículas mistas apresentaram valores médios semelhantes ou inferiores aos obtidos para os painéis OSB e superiores ou semelhantes aos atingidos para os painéis MDP.

Neste estudo, de forma geral, os painéis estruturais de partículas mistas apresentaram valores médios próximos aos obtidos para OSB e melhores que os obtidos para MDP. Este fato confirma a qualidade técnica desse tipo de painel, pois além de apresentar propriedades físicas e mecânicas adequadas para a produção de painéis com fins estruturais, é produzido com camada externa de melhor acabamento superficial em relação aos painéis OSB, o que facilita a aplicação de revestimentos, muito importante para determinadas aplicações.

Em relação aos requisitos normativos, para o módulo de elasticidade, apenas os painéis OSB de Pinus spp. nos sentidos paralelo e perpendicular e os painéis OSB de Eucalyptus badjensis no sentido paralelo atenderam aos requisitos estabelecidos pela norma CSA 0437. 
Com relação às normas europeias, todos os tratamentos atenderam aos requisitos mínimos estabelecidos pela EN 312 e EN 300 para o sentido perpendicular. Por outro lado, somente os painéis OSB de Pinus spp. e Eucalyptus badjensis atenderam aos requisitos mínimos da EN 300 para o sentido paralelo.

Para o módulo de ruptura, todos os tratamentos atingiram os valores mínimos estabelecidos pela norma EN 312. Comparando com à norma CSA 0437, apenas o MDP de Eucalyptus badjensis apresentou valor médio inferior ao referido valor.

Em relação à norma EN 300, todas as composições atingiram os valores médios estabelecidos para o sentido perpendicular. Já, para o sentido paralelo, não atenderam aos requisitos normativos, os painéis MDP e os painéis de partículas mistas, nas composições 20/60/20 para as duas espécies e 15/70/15 para os painéis Eucalyptus badjensis na camada externa.

Para o ensaio de tração perpendicular à superfície, todos os tratamentos apresentaram valores médios em conformidade com os requisitos normativos da CSA 0437, EN 300 e EN 312.

Os painéis produzidos com Eucalyptus badjensis e Pinus spp. na camada externa foram semelhantes estatisticamente para o módulo de elasticidade, o módulo de ruptura e a tração perpendicular à superfície (Tabela 8).

Tabela 8. Valores médios de módulo de elasticidade, módulo de ruptura e tração perpendicular à superfície para painéis com diferentes espécies na camada externa

\begin{tabular}{cccc}
\hline Espécie & MOE $(\mathbf{M P a})$ & MOR $(\mathrm{MPa})$ & TP $(\mathbf{M P a})$ \\
\hline Pinus spp. & $2570,82 \mathrm{a}$ & $22,92 \mathrm{a}$ & $0,51 \mathrm{a}$ \\
& $(23,99)$ & $(25,13)$ & $(13,66)$ \\
E. bad & $2557,62 \mathrm{a}$ & $20,90 \mathrm{a}$ & $0,53 \mathrm{a}$ \\
CSA0437* & $(25,62)$ & $(27,94)$ & $(16,74)$ \\
EN312 & 3040 & 16,87 & 0,34 \\
EN300 paralelo** & 1800 & 13,00 & 0,40 \\
EN300 perpendicular** & 3500 & 20,00 & 0,32 \\
\hline
\end{tabular}

Médias seguidas de letras iguais na mesma coluna não diferem estatisticamente entre si pelo teste de Tukey ao nível de $95 \%$ de probabilidade. Coeficiente de variação, em porcentagem, entre parênteses. MOE: Módulo de elasticidade; MOR: Módulo de ruptura; TP: Tração perpendicular à superfície, E bad: Eucalyptus badjensis. *Categoria R1: Painéis com partículas dispostas de forma aleatórias, **OSB 3: Painéis estruturais para uso em condições úmidas.

Para resistência à flexão estática, a semelhança estatística pode ser atribuída à pequena diferença na massa especifica das madeiras de Eucalyptus badjensis e Pinus spp. utilizadas e, consequentemente, pequena variação na razão de compactação para painéis produzidos com a mesma massa específica nominal. Os dados apresentados na Tabela 2, corroboram com esse entendimento, uma vez que a maior diferença de razão de compactação entre painéis de partículas mistas de mesma massa específica nominal foi de 0,145.

Para tração perpendicular à superfície, a semelhança estatística pode ser atribuída ao fato de a camada interna destes painéis ter sido produzida com partículas do tipo strand de Eucalyptus badjensis para ambos os casos e, desse modo, ambos os painéis terem sido submetidos aos mesmos esforços de tração.

Na comparação com os requisitos normativos, para o módulo de elasticidade, tanto os painéis de Eucalyptus badjensis, quanto os de Pinus spp. na camada externa, atenderam aos requisitos mínimos da norma EN 312 e EN 300 para sentido perpendicular. Já, para o sentido paralelo, os resultados obtidos foram inferiores aos valores mínimos estabelecidos pela norma EN 300. 
Para o módulo de ruptura e a tração perpendicular à superfície, os valores médios obtidos tanto para Eucalyptus badjensis, quanto para Pinus spp. atenderam aos requisitos normativos da EN 300, EN 312 e CSA 0437.

Tanto para o módulo de elasticidade, quanto para o módulo de ruptura, os painéis produzidos com a composição sliver/strand/sliver de 10/80/10 apresentaram valores médios maiores que os painéis com menor proporção de partículas strand na camada interna (Tabela 9). Resultado este, que comprova a influência do comprimento das partículas nas propriedades de flexão estática, uma vez que, painéis com maior quantidade de partículas do tipo strand apresentaram os maiores valores.

Tabela 9. Valores médios de módulo de elasticidade, módulo de ruptura e tração perpendicular para painéis produzidos com diferentes composições de camadas

\begin{tabular}{cccc}
\hline Composição & MOE (MPa) & MOR (MPa) & TP (MPa) \\
\hline PEPM & $231^{\prime} 6,02 \mathrm{~b}$ & $19,64 \mathrm{~b}$ & $0,51 \mathrm{a}$ \\
$\mathbf{2 0 / 6 0 / 2 0}$ & $(12,62)$ & $(12,49)$ & $(14,85)$ \\
PEPM & $2495,10 \mathrm{~b}$ & $21,50 \mathrm{~b}$ & $0,53 \mathrm{a}$ \\
$\mathbf{1 5 / 7 0 / 1 5}$ & $(15,06)$ & $(22,03)$ & $(10,04)$ \\
PEPM & $2895,63 \mathrm{a}$ & $24,75 \mathrm{a}$ & $0,51 \mathrm{a}$ \\
$\mathbf{1 0 / 8 0 / 1 0}$ & $(21,78)$ & $(21,10)$ & $(19,57)$ \\
CSA0437* & 3040 & 16,87 & 0,34 \\
EN312 & 1800 & 13,00 & 0,40 \\
EN300 paralelo** & 3500 & 20,00 & 0,32 \\
EN300 perpendicular** & 1500 & 10,00 &
\end{tabular}

Médias seguidas de letras iguais na mesma coluna não diferem estatisticamente entre si pelo teste de Tukey ao nível de 95\% de probabilidade. Coeficiente de variação, em porcentagem, entre parênteses. MOE: Módulo de elasticidade; MOR: Módulo de ruptura; TP: Tração perpendicular à superfície. PEPM: Painel estrutural de partículas mistas. ${ }^{*}$ Categoria R1: Painéis com partículas dispostas de forma aleatórias, **OSB 3: Painéis estruturais para uso em condições úmidas.

Para o ensaio de tração perpendicular à superfície, todas as composições foram semelhantes estatisticamente.

Este comportamento pode ser explicado pelo fato de as camadas internas terem sido formadas com o mesmo material e, por conseguinte, serem submetidas as mesmas tensões de tração.

Em comparação com os requisitos normativos, para o módulo de elasticidade, todas as composições atenderam aos requisitos mínimos da norma EN 312 e EN 300 no sentido perpendicular, e obtiveram valores inferiores aos estabelecidos pela CSA 0437 e EN 312 no sentido perpendicular.

Para o módulo de ruptura, todas as composições atingiram os valores estabelecidos pelas normas CSA 0437, EN 312 e EN 300 no sentido perpendicular. Para a EN 300 no sentido paralelo apenas a composição 20/60/10 apresentou valores inferiores aos estabelecidos.

Para a tração perpendicular à superfície, todas as composições atenderam aos requisitos normativos estabelecidos pela EN 300, EN 312 e CSA 0437.

Os resultados obtidos para as propriedades mecânicas demonstram a qualidade técnica dos painéis estruturais de partículas mistas, uma vez que, de forma geral, apresentaram maiores valores médios em relação aos painéis MDP e próximos aos encontrados para OSB, tendo como diferencial o melhor acabamento superficial proporcionado pela utilização de camada externa de MDP e a maior uniformidade nas propriedades nos diferentes sentidos do painel, em função de ser produzido com partículas dispostas de forma aleatória. 


\section{CONCLUSÕES}

- Os painéis de partículas mistas apresentam potencial para fins estruturais, uma vez que atendem aos requisitos normativos para a maior parte das propriedades avaliadas.

- A composição de painel com $10 \%$ de partículas do tipo sliver nas camadas externas e $80 \%$ de partículas do tipo strand na camada interna (10/80/10) é a que apresenta os melhores resultados, com as médias estatisticamente semelhantes ou superiores às demais composições para todas as propriedades analisadas.

- A madeira de Eucalyptus badjensis, bem como, sua utilização em conjunto com partículas de Pinus spp. nas camadas externas, possuem potencial para produção de painéis estruturais de partículas mistas, tendo em vista que atenderam aos requisitos normativos para a maioria das propriedades.

\section{REFERÊNCIAS}

Associação Brasileira de Normas Técnicas - ABNT. (1997). NBR 7190: projeto de estruturas de madeira (107 p.). Rio de Janeiro.

Associação Brasileira de Normas Técnicas - ABNT. (2006). NBR 14810: chapas de madeira aglomerada (51 p.). Rio de Janeiro.

Canadian Standards Association - CSA. (1993). CSA 0437.0: OSB and Waferboard (18 p.). Ontario.

Chiromito, E. M. S., Campos, C. I., Ferreira, B. S., Christoforo, A. L., \& Lahr, F. A. R. (2016). Propriedades mecânicas de painéis produzidos com lascas de madeira em três diferentes comprimentos. Scientia Forestalis, 44(109), http://dx.doi.org/10.18671/scifor.v44n109.17.

European Committee for Standardization. (1993b). EN 323: determination of density. English version. London: CEN.

European Committee for Standardization. (2002a). EN 310: determination of modulus of elasticity in bending and of bending strength. London: CEN.

European Committee for Standardization. (2002b). EN 317: determination of swelling in thickness after immersion in water. London: CEN.

European Committee for Standardization. (2002c). EN 319: determinação da resistência à tração perpendicular às faces da placa. London: CEN.

European Committee for Standardization. (2003). EN 312: particleboards: specifications. London: CEN.

European Committee for Standardization. (2006). EN 300: Oriented Strand Boards (OSB): definitions, classification and specifications. London: CEN.

Geimer, R. L., \& Price, E. W. (1978). Construction variables considered in the fabrication of structural flakeboard (WO General Technical Report, No. 5, pp. 69-80). Washington: USDA Forest Service.

Higa, R. C. V., Higa, A. R., \& Alves, E. C. (2002). Comportamento de progênies de Eucalyptus badjensis Beuzev. \& Welch em dois locais da Região Sul do Brasil (Boletim de Pesquisa Florestal, No. 45). Colombo: Embrapa Florestas.

Iwakiri, S. (2005). Painéis de madeira. Curitiba: FUPEF.

Iwakiri, S., Vianez, B. F., Weber, C., Trianoski, R., \& Almeida, V. C. (2012). Avaliação das propriedades de painéis aglomerados produzidos com resíduos de serrarias de nove espécies da Amazônia. Acta Amazonica, 42(1), 59-64. http://dx.doi.org/10.1590/S0044-59672012000100007.

Maloney, T. M. (1993). Modern particleboard and dry-process fiberboard manufacturing (2nd ed.). São Francisco: Miller Freeman. 689 p.

Marzbani, P., Afrouzi, Y. M., \& Omidvar, A. (2015). The effect of nano-zinc oxide on particleboard decay resistance. Maderas. Ciencia y Tecnología, 17(1), 63-68. http://dx.doi.org/10.4067/S0718221X2015005000007.

Moslemi, A. A. (1974). Particleboard (Vol. 2). Illionis: Southern Illionis University. 245 p.

Pereira, G. F., Rios, P. D. A., Buss, R., Vieira, H. C., Grubert, W., Cunha, A. B., \& Belini, U. L. (2017). Painéis de madeira aglomerada de Eucalyptus badjensis e Pinus spp. Scientia Forestalis, 45(114), 373-382. http://dx.doi.org/10.18671/scifor.v45n114.13.

Thoemen, H., Irle, M., \& Sernek, M. (2010). Wood-based panel an introduction for specialists. London: Brunel University Press. 
Trianoski, R., Iwakiri, S., \& Chies, D. (2016). Utilização da madeira de Cryptomeria japônica para produção de painéis de partículas orientadas. Scientia Forestalis, 44(110), 487-496. http://dx.doi.org/10.18671/scifor.v44n110.21.

Contribuição dos Autores: GFP: Conceituação, Curadoria de Dados, Análise Formal, Investigação, Metodologia, Escrita - Primeira Redação, Escrita - Revisão e Edição. SI: Conceituação, Metodologia, Administração do Projeto, Recursos, Validação, Escrita - Revisão e Edição. RT: Conceituação, Metodologia, Administração do Projeto,

Recursos, Supervisão, Escrita - Revisão e Edição. PDR: Conceituação, Metodologia, Recursos, Validação, Escrita Revisão e Edição. RZR: Conceituação, Curadoria de Dados, Análise Formal, Escrita - Revisão e Edição. 